\title{
Brain Mapping Using Neuroimaging
}

\author{
Woo-Suk Tae ${ }^{1}$, Shin-Hyuk Kang ${ }^{1,2}$, Byung-Joo Ham ${ }^{1,3}$, Byung-Jo Kim ${ }^{1,4}$, Sung-Bom Pyun ${ }^{1,5, *}$ \\ ${ }^{1}$ Brain Convergence Research Center, Korea University, Seoul 02841, Korea \\ ${ }^{2}$ Department of Neurosurgery, Korea University College of Medicine, Seoul 02841, Korea \\ ${ }^{3}$ Department of Psychiatry, Korea University College of Medicine, Seoul 02841, Korea \\ ${ }^{4}$ Department of Neurology, Korea University College of Medicine, Seoul 02841, Korea \\ ${ }^{5}$ Department of Physical Medicine and Rehabilitation, Korea University College of Medicine, Seoul 02841, Korea
}

*Correspondence to:

Pyun SB,

Tel: +82-2-920-6480

Fax: +82-2-929-9951

E-mail: rmpyun@korea.ac.kr

Received December 25, 2016

Revised December 28, 2016

Accepted December 28, 2016

\begin{abstract}
Mapping brain structural and functional connections through the whole brain is essential for understanding brain mechanisms and the physiological bases of brain diseases. Although region specific structural or functional deficits cause brain diseases, the changes of interregional connections could also be important factors of brain diseases. This review will introduce common neuroimaging modalities, including structural magnetic resonance imaging (MRI), functional MRI (fMRI), diffusion tensor imaging, and other recent neuroimaging analyses methods, such as voxel-based morphometry, cortical thickness analysis, local gyrification index, and shape analysis for structural imaging. Tract-Based Spatial Statistics, TRActs Constrained by UnderLying Anatomy for diffusion MRI, and independent component analysis for $\mathrm{fMRI}$ also will also be introduced.
\end{abstract}

Key Words: Brain connectome, Magnetic resonance imaging, Diffusion tensor imaging, Functional magnetic resonance imaging, Brain mapping

\section{INTRODUCTION}

MRI has been widely used in hospitals for disease diagnosis, staging and follow-up, which has the advantage of avoiding needlessly the body to ionizing radiation. Magnetic resonance imaging (MRI) is playing a crucial role in the study of neuropsychiatric diseases, and the usefulness of MRI has been rapidly increased. MRI delineates the structural and functional alterations determined by disease conditions. Modern MRI technologies are of great interest due to MRI's potential to characterize the signature of each neurodegenerative process and help both the diagnostic aspect and the monitoring of disease development (Agosta et al., 2016).

Structural MRI has become the standard for routine clinical procedure; it shows anatomical detail with high sensitivity to pathology (Mak et al., 2016). Relatively new quantitative measures of diffusion tensor imaging (DTI) and fMRI are commonly used for research and clinical purposes and will soon become standard clinical tools (Christidi et al., 2016;
Hu et al., 2016). Recently, DTI technique has advanced to the point that it has very high resolution with pathology specific details. It has revealed microstructural changes in the axon and myelin of brain white matter. Functional MRI (fMRI), on the other hand, measures brain activity indirectly; Task specific or disease related intrinsic network of brain area could be identifiable.

In this review paper, we will introduce common MRI based neuroimaging modalities and analysis techniques. To understand the strengths and limitations of each neuroimaging analysis, the details of the imaging characteristics for each technique will be explained.

\section{NEUROIMAGING MODALITIES}

Structural T1-weighted MRI provides a variety of information, including the integrity of gray and white matter, and both quantitative and qualitative shapes and size of substructures. Using the recent clinical submillimeter high resolu-

(a) This is an open-access article distributed under the terms of the Creative Commons Attribution Non-Commercial License (http://creativecommons.org/licenses/by-nc/4.0) which permits unrestricted noncommercial use, distribution, and reproduction in any medium, provided the original work is properly cited.

Copyrights @ 2016 by Korean Society of Microscopy 
tion clinical MRIs (Fig. 1), structural MRI has been used not only for visual diagnosis purposes, but also for the production of raw data in the neuroimaging of brain volumetry, voxel-

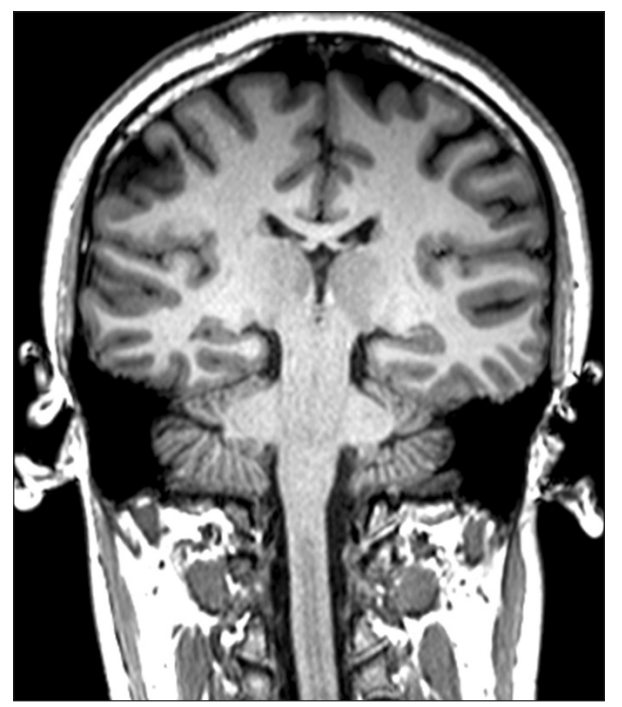

Fig. 1. Three dimensional T1-weighted 3 Tesla magnetic resonance imaging with $0.5 \mathrm{~mm}$ isotropic resolution shows very detailed brain anatomy, and it clearly shows habenula nuclei in the bilateral medial thalamic areas with high signal intensity. based morphometry (VBM), cortical thickness analysis, shape analysis, and the local gyrification index (LGI) (Fig. 2). These image processing techniques provide unique quantitative indices and clinical information, and these advanced methods have been adopted for clinical and neuroscience research (Thompson et al., 2014).

Another major area of interest in current neuroimaging research is the tracking of functional networks and pathways in the brains. Whereas functional localization and the connectivity in gray matter have been extensively investigated using fMRI, structural pathways within white matter have been studied primarily with DTI and fiber tractography. DTI is a relatively recent MRI technique that measures the direction and magnitude of the motility of water molecules in tissue. It has made it possible to determine the orientation and diffusion characteristics of white matter and has revealed microstructural changes in the brain. Fractional anisotropy (FA) is the most commonly used DTI scalars, but axial diffusivity (AD), radial diffusivity (RD), mean diffusivity (MD) also have important clinical usefulness. FA is related to and highly sensitive to microstructural fiber integrity, whereas $\mathrm{AD}$ is related to axonal injury. When axons are damaged FA decreased, whereas $\mathrm{AD}$ increases with brain maturation. $\mathrm{RD}$ increases in myelin-damaged white matter tissue; $\mathrm{RD}$ values are also influenced by axonal diameters and densities.

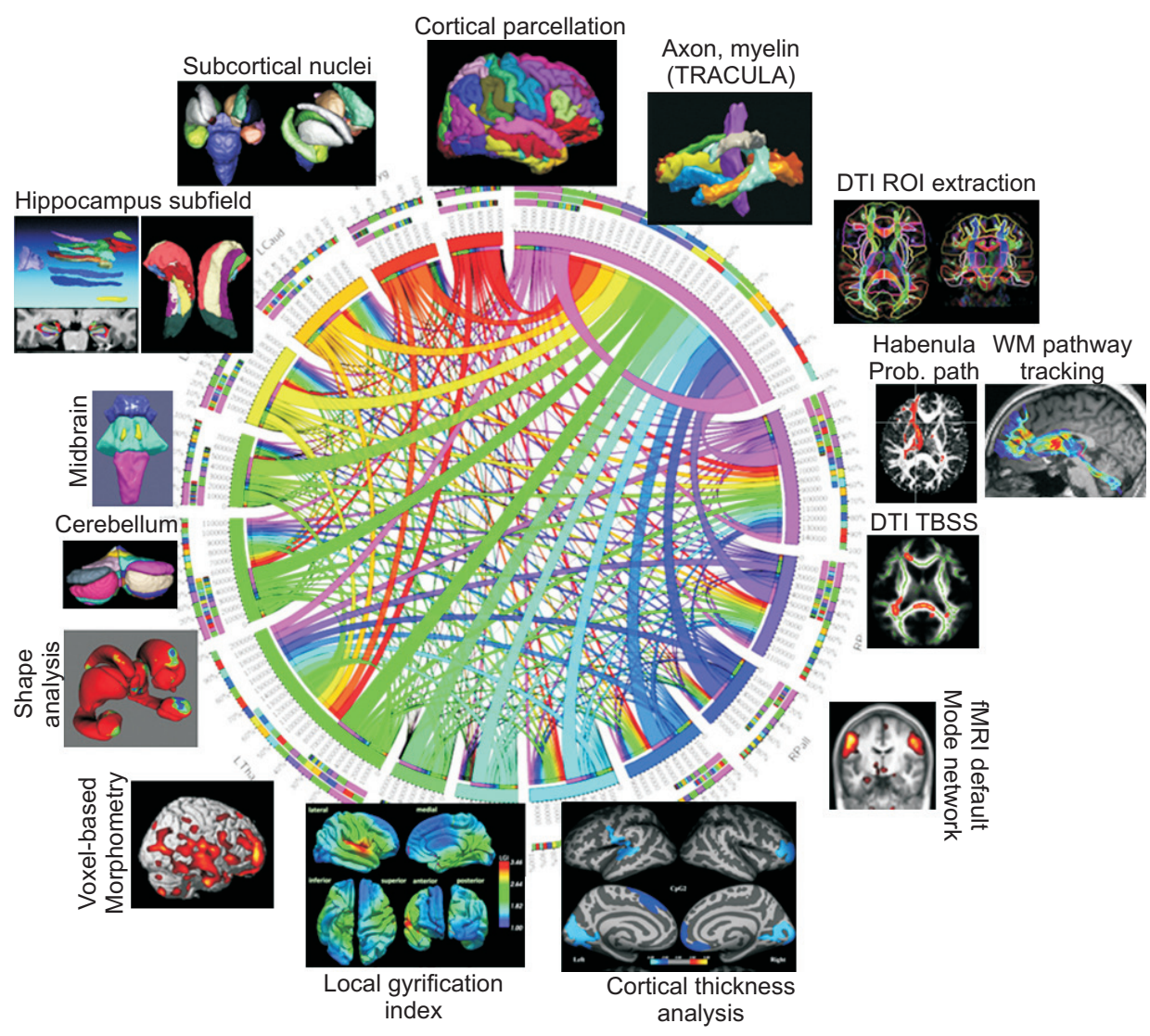

Fig. 2. This figure illustrates various brain mapping methods for structural and functional mapping. The center image is a connectogram describing connectional strength among brain subregions. TRACULA, TRActs Constrained by UnderLying Anatomy; DTI, diffusion tensor imaging; ROI, region of interest; Prob., probtrack; WM, white matter; TBSS, Tract-Based Spatial Statistics; fMRI, functional magnetic resonance imaging. 
Although FA is highly sensitive to microstructural changes, it is not very specific to the type of changes. Therefore, to maximize the utility of DTI, the use of multiple DTI scalars ( $\mathrm{AD}, \mathrm{RD}$, and $\mathrm{MD})$ is highly recommended to better characterize the tissue microstructure (Alexander et al., 2007). fMRI measures brain activity indirectly by detecting blood flow associated blood-oxygen-level dependent (BOLD) contrast changes (Ogawa, 2012). If a task specific brain area is active, the areas related to the task consume more oxygen and to compensate this increased oxygen demand blood flow increases to the active brain regions. By statistical modeling, fMRI is used to generate BOLD activation maps showing the brain regions involved in particular tasks processes. Whereas functional localization and the connectivity in gray matter have been investigated using fMRI structural pathways within white matter have been studied primarily with DTI and fiber tractography. However, DTI tractography is limited to the structural pathway only, and does not provide any information regarding the functional dynamics in the white matter. A recent study demonstrated the auditory functional pathways in white and gray matter using fMRI. This study suggested that direct evaluation of central auditory functional networks and pathways in the human brain is possible using BOLD fMRI (Tae et al., 2014).

\section{NEUROIMAGE ANALYSIS TECHNIQUES}

MRI could provide information about structural characteristics such as the sub-regional volume, shape, sulcal depth, and tissue concentrations of human brains. VBM is an unbiased whole brain approach for detecting structural differences in gray or white matter between different populations (Good et al., 2001). VBM also provides a systematic estimate of regional gray matter based on voxel-by-voxel comparison through the whole brain and has been used to measure the regional tissue concentration and volume changes in MRI. The high-dimensional warping algorithm called "Diffeomorphic Anatomical Registration Through Exponentiated Lie Algebra" provides high spatial registration accuracy, the most important factor for obtaining optimal voxel-wise structural analyses (Ashburner, 2007).

Cortical thickness analysis processed at the nodes of a three dimensional polygonal mesh has been found to offer the advantage of providing a direct quantitative index of cortical morphology. It has the benefit of providing a quantitative morphological index of brain cortices. Cortical thickness measured from cortical surfaces differentiates cortices of opposing sulcal walls within the same sulcal bed, enabling more precise measurement in deep sulci and the analysis of morphology as a cortical sheet. This method measures cortical gray matter thickness, whereas VBM measures regional tissue concentrations or volume changes of gray or white matter. The LGI reflects the pattern and degree of cortical folding, some studies have reported that abnormal gyrification could be a more stable biomarker of brain disease than other neuroanatomical indices due to its putative state independence. The LGI can be measured with an automatically reconstructed cortical surface model (Nenadic et al., 2015). The LGI can quantify the early neural development of cortical connectivity in which the fiber tension of densely connected cortical regions forms gyri and sparsely connected regions move apart to become separated by sulci during the second trimester of pregnancy (Nanda et al., 2014). Abnormal gyrification is thought to reflect damaged structural or functional connectivity of the brain cortex (Nixon et al., 2014). Recent research has suggested that the LGI could describe a useful endophenotype of several neuropsychiatric disorders including schizophrenia, bipolar disorder, and schizoaffective disorder (Janssen et al., 2014).

Shape analysis is a method for the statistical evaluation of morphometric changes of brain sub-regions. Brain subregional volumetry provides the measured volume information as a mass, and though volumetry could be advantageous in the detection of mild mass changes throughout the brain

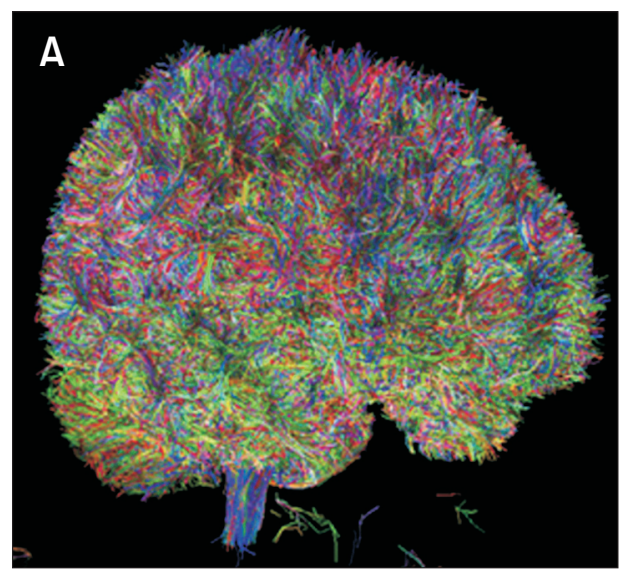

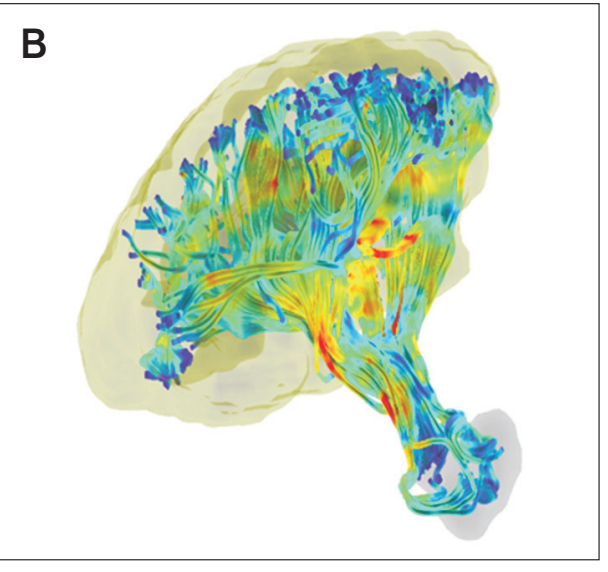

Fig. 3. White matter fiber reconstruction using diffusion tensor imaging. Whole brain white matter reconstructed with 64 direction diffusion tensor imaging (A), and brain fibers passing through cerebellar vermis and frontal lobe showing very dense structural connectivity (B). 
sub-regional nuclei, it might also be valuable to screen the atrophic or hypertrophic sub-regions. Shape analysis allows the statistical assessment of the sub-regional anatomy of morphologically changed areas, with 3D modeling of the subregions so that contracted or expanded sub-regions can be identified. Note that, shape analysis is a more local change specific method, rather than a whole brain technique.

Voxel-wise statistical analyses of the FA, AD, RD, and MD data estimated from DTI could be carried out using TractBased Spatial Statistics (TBSS) (Smith et al., 2006). FA images were created by fitting a tensor model to the raw diffusion data using FMRIB's diffusion toolbox, and a brain image was extracted. All FA data were aligned into a common space using the FMRIB's nonlinear image registration tool. Subsequently, the mean FA image of all subjects was generated and thinned to create a mean FA skeleton image that describes the centers of all white matter tracts. Each subject's FA data were projected onto the new mean FA skeleton image, and the voxel-wise statistical calculation was tested and the results were displayed on the mean FA skeleton. TBSS has the advantage of correcting white matter tracks' spatial alignment problem. TRActs Constrained by UnderLying Anatomy (TRACULA) is a fiber tracking method with automated probabilistic reconstruction of 18 major white matter pathways from DTI (Yendiki et al., 2011). It uses prior information of the white matter pathways from a set of training subject which was previously built. It gives information on the specific tract involved in a brain disease and the various types of white matter defects (Fig. 3).

Independent component analysis (ICA) is a blind sourceseparation method that is commonly used for fMRI analysis. ICA identifies functional brain networks, and it extracts unknown source BOLD signals from the known mix of signals. The commonly used spatial ICA decomposes time series imaging into a set of linearly separable, spatially independent components and their associated time courses
(McKeown et al., 1998; McKeown \& Sejnowski, 1998). One of the problems with hypothesis-driven statistical methods, such as a general linear model, is the difficulty in modeling real neural activity from various artifactual MRI signals. ICA can, however, separate real brain networks from various noise sources with any prior MRI signal information. The major advantage of ICA is that it is data-driven approach that does not require an a priori response modeling of neural activity. This allows the avoidance of bias towards predicted brain behavior, unlike traditional model-driven analyses.

\section{CONCLUSIONS}

Structural MRI has become the standard for routine clinical investigations of the brain; it offers anatomical detail and is highly sensitive to pathological abnormalities. Relatively new quantitative measures of DTI and fMRI are being commonly used for research and clinical purposes, and will soon become standard clinical tools. In this short review paper, we presented a comprehensive survey of neuroimaging modalities and analysis techniques. These recent neuroimaging methods have important applications in clinical and basic neuroscience research. The understanding of the individual characteristics of imaging modalities and analysis methods is prerequisite condition.

\section{CONFLICT OF INTEREST}

No potential conflict of interest relevant to this article was reported.

\section{ACKNOWLEDGMENTS}

This work was supported by the National Research Foundation of Korea (NRF) funded by the Korea government (MSIP) (No. 2016R1A2B4009206).

\section{REFERENCES}

Agosta F, Galantucci S, and Filippi M (2016) Advanced magnetic resonance imaging of neurodegenerative diseases. Neurol. Sci. doi: 10.1007/s10072-016-2764-X.

Alexander A L, Lee J E, Lazar M, and Field A S (2007) Diffusion tensor imaging of the brain. Neurotherapeutics 4, 316-329.

Ashburner J (2007) A fast diffeomorphic image registration algorithm. Neuroimage 38, 95-113.

Christidi F, Karavasilis E, Samiotis K, Bisdas S, and Papanikolaou N (2016) Fiber tracking: a qualitative and quantitative comparison between four different software tools on the reconstruction of major white matter tracts. Eur. J. Radiol. Open. 3, 153-161.
Good C D, Johnsrude I S, Ashburner J, Henson R N, Friston K J, and Frackowiak R S (2001) A voxel-based morphometric study of ageing in 465 normal adult human brains. Neuroimage 14, 21-36.

Hu M L, Zong X F, Mann J J, Zheng J J, Liao Y H, Li Z C, He Y, Chen X G, and Tang J S (2016) A review of the functional and anatomical default mode network in schizophrenia. Neurosci. Bull. doi: 10.1007/ s12264-016-0090-1.

Janssen J, Aleman-Gomez Y, Schnack H, Balaban E, Pina-Camacho L, Alfaro-Almagro F, Castro-Fornieles J, Otero S, Baeza I, Moreno D, Bargalló N, Parellada M, Arango C, and Desco M (2014) Cortical morphology of adolescents with bipolar disorder and with schizophrenia. Schizophr. Res. 158, 91-99. 
Mak E, Gabel S, Mirette H, Su L, Williams G B, Waldman A, Wells K, Ritchie K, Ritchie C, and O'Brien J (2016) Structural neuroimaging in preclinical dementia: from microstructural deficits and grey matter atrophy to macroscale connectomic changes. Ageing Res. Rev. S1568-1637, 30062-30069.

McKeown M J, Makeig S, Brown G G, Jung T P, Kindermann S S, Bell A J, and Sejnowski T J (1998) Analysis of $\mathrm{fMRI}$ data by blind separation into independent spatial components. Hum. Brain Mapp. 6, 160188.

Mckeown M J and Sejnowski T J (1998) Independent component analysis of fMRI data: examining the assumptions. Hum. Brain Mapp. 6, 368372.

Nanda P, Tandon N, Mathew I T, Giakoumatos C I, Abhishekh H A, Clementz B A, Pearlson G D, Sweeney J, Tamminga C A, and Keshavan M S (2014) Local gyrification index in probands with psychotic disorders and their first-degree relatives. Biol. Psychiatry 76, 447-455

Nenadic I, Maitra R, Dietzek M, Langbein K, Smesny S, Sauer H, and Gaser C (2015) Prefrontal gyrification in psychotic bipolar I disorder vs. schizophrenia. J. Affect Disord. 185, 104-107.

Nixon N L, Liddle P F, Nixon E, Worwood G, Liotti M, and Palaniyappan L (2014) Biological vulnerability to depression: linked structural and functional brain network findings. Br. J. Psychiatry 204, 283-289.

Ogawa S (2012) Finding the BOLD effect in brain images. Neuroimage 62, 608-609.

Smith S M, Jenkinson M, Johansen-Berg H, Rueckert D, Nichols T E, Mackay C E, Watkins K E, Ciccarelli O, Cader M Z, Matthews P M, and Behrens T E (2006) Tract-based spatial statistics: voxelwise analysis of multi-subject diffusion data. Neuroimage 31, 1487-1505.

Tae W S, Yakunina N, Kim T S, Kim S S, and Nam E C (2014) Activation of auditory white matter tracts as revealed by functional magnetic resonance imaging. Neuroradiology 56, 597-605.

Thompson P M, Stein J L, Medland S E, Hibar D P, Vasquez A A, Renteria M E, Toro R, Jahanshad N, Schumann G, Franke B, Wright M J, Martin N G, Agartz I, Alda M, Alhusaini S, Almasy L, Almeida J, Alpert K, Andreasen N C, Andreassen O A, Apostolova L G, Appel K, Armstrong $\mathrm{N} J$, Aribisala B, Bastin M E, Bauer M, Bearden C E, Bergmann O, Binder E B, Blangero J, Bockholt H J, Bøen E, Bois C, Boomsma D I, Booth T, Bowman I J, Bralten J, Brouwer R M, Brunner H G, Brohawn D G, Buckner R L, Buitelaar J, Bulayeva K, Bustillo J R, Calhoun V D, Cannon D M, Cantor R M, Carless M A, Caseras X, Cavalleri G L, Chakravarty M M, Chang K D, Ching C R, Christoforou A, Cichon S, Clark V P, Conrod P, Coppola G, Crespo-Facorro B, Curran J E, Czisch M, Deary I J, de Geus E J, den Braber A, Delvecchio G, Depondt C, de Haan L, de Zubicaray G I, Dima D, Dimitrova R, Djurovic S, Dong H,
Donohoe G, Duggirala R, Dyer T D, Ehrlich S, Ekman C J, Elvsåshagen T, Emsell L, Erk S, Espeseth T, Fagerness J, Fears S, Fedko I, Fernández G, Fisher S E, Foroud T, Fox P T, Francks C, Frangou S, Frey E M, Frodl T, Frouin V, Garavan H, Giddaluru S, Glahn D C, Godlewska B, Goldstein R Z, Gollub R L, Grabe H J, Grimm O, Gruber O, Guadalupe T, Gur R E, Gur R C, Göring H H, Hagenaars S, Hajek T, Hall G B, Hall J, Hardy J, Hartman C A, Hass J, Hatton S N, Haukvik U K, Hegenscheid K, Heinz A, Hickie I B, Ho B C, Hoehn D, Hoekstra P J, Hollinshead M, Holmes A J, Homuth G, Hoogman M, Hong L E, Hosten N, Hottenga J J, Hulshoff Pol H E, Hwang K S, Jack C R Jr, Jenkinson M, Johnston C, Jönsson E G, Kahn R S, Kasperaviciute D, Kelly S, Kim S, Kochunov P, Koenders L, Krämer B, Kwok J B, Lagopoulos J, Laje G, Landen M, Landman B A, Lauriello J, Lawrie S M, Lee P H, Le Hellard S, Lemaître H, Leonardo C D, Li C S, Liberg B, Liewald D C, Liu X, Lopez L M, Loth E, Lourdusamy A, Luciano M, Macciardi F, Machielsen M W, Macqueen G M, Malt U F, Mandl R, Manoach D S, Martinot J L, Matarin M, Mather K A, Mattheisen M, Mattingsdal M, Meyer-Lindenberg A, McDonald C, Mclntosh A M, McMahon F J, McMahon K L, Meisenzahl E, Melle I, Milaneschi Y, Mohnke S, Montgomery G W, Morris D W, Moses E K, Mueller B A, Muñoz Maniega S, Mühleisen T W, MüllerMyhsok B, Mwangi B, Nauck M, Nho K, Nichols T E, Nilsson L G, Nugent A C, Nyberg L, Olvera R L, Oosterlaan J, Ophoff R A, Pandolfo M, Papalampropoulou-Tsiridou M, Papmeyer M, Paus T, Pausova Z, Pearlson G D, Penninx B W, Peterson C P, Pfennig A, Phillips M, Pike G B, Poline J B, Potkin S G, Pütz B, Ramasamy A, Rasmussen J, Rietschel M, Rijpkema M, Risacher S L, Roffman J L, Roiz-Santiañez R, Romanczuk-Seiferth N, Rose E J, Royle N A, Rujescu D, Ryten M, Sachdev P S, Salami A, Satterthwaite T D, Savitz J, Saykin A J, Scanlon C, Schmaal L, Schnack H G, Schork A J, Schulz S C, Schür R, Seidman L, Shen L, Shoemaker J M, Simmons A, Sisodiya S M, Smith C, Smoller J W, Soares J C, Sponheim S R, Sprooten E, Starr J M, Steen V M, Strakowski S, Strike L, Sussmann J, Sämann P G, Teumer A, Toga A W, Tordesillas-Gutierrez D, Trabzuni D, Trost S, Turner J, Van den Heuvel M, van der Wee N J, van Eijk K, van Erp T G, van Haren N E, van’t Ent D, van Tol M J, Valdés Hernández M C, Veltman D J, Versace A, Völzke $H$, Walker R, Walter $H$, Wang L, Wardlaw J M, Weale M E, Weiner M W, Wen W, Westlye L T, Whalley H C, Whelan C D, White T, Winkler A M, Wittfeld K, Woldehawariat G, Wolf C, Zilles D, Zwiers M P, Thalamuthu A, Schofield P R, Freimer N B, Lawrence N S, Drevets W; Alzheimer's Disease Neuroimaging Initiative, EPIGEN Consortium, IMAGEN Consortium, Saguenay Youth Study (SYS) Group (2014) The ENIGMA Consortium: large-scale collaborative analyses of neuroimaging and genetic data. Brain Imaging Behav. 8, 153-182.

Yendiki A, Panneck P, Srinivasan P, Stevens A, Zöllei L, Augustinack J, Wang R, Salat D, Ehrlich S, Behrens T, Jbabdi S, Gollub R, and Fischl B (2011) Automated probabilistic reconstruction of white-matter pathways in health and disease using an atlas of the underlying anatomy. Front Neuroinform 5, 23. 